\title{
Recurrence and disease-free survival outcomes after computed tomography-guided needle biopsy in stage IA non-small cell lung cancer patients in China: a propensity score matching analysis
}

\author{
Chuang $\mathrm{He}^{1 \#}$, Hualong $\mathrm{Yu}^{2 \#}$, Changyi $\mathrm{Li}^{3}$, Xin Zhang ${ }^{4}$, Zhicheng Huang ${ }^{5}$, Mingyang Liu ${ }^{6}$, Lunbing Tong ${ }^{7}$, \\ Jun Zhu ${ }^{8}$, Wei $\mathrm{Wu}^{9}$, Xuequan Huang ${ }^{1}$ \\ ${ }^{1}$ Treatment Center of Minimally Invasive Intervention and Radioactive Particles, First Affiliated Hospital of the Army Medical University, \\ Chongqing, China; ${ }^{2}$ Department of Radiology, Affiliated Hospital of Qingdao University, Qingdao, China; ${ }^{3}$ Department of Respiratory, The Second \\ Affiliated Hospital of Chongqing Medical University, Chongqing, China; ${ }^{4}$ Department of Thoracic Surgery, General Hospital of Heilongjiang \\ Province Land Reclamation Bureau, Harbin, China; ${ }^{5}$ Department of Radiology, Jilin Provincial Cancer Hospital, Changchun, China; ${ }^{6}$ Department of \\ Oncology, General Hospital of Heilongjiang Province Land Reclamation Bureau, Harbin, China; ${ }^{7}$ Department of Radiology, Sichuan Police Force \\ Hospital, Leshan, China; ${ }^{8}$ Department of Radiology, The Second People's Hospital of Yibin, Yibin, China; ${ }^{9}$ Department of Thoracic Surgery, First \\ Affiliated Hospital of the Army Medical University, Chongqing, China
}

"These authors contributed equally to this work.

Correspondence to: Wei Wu. Department of Thoracic Surgery, First Affiliated Hospital of the Army Medical University, Chongqing 400038, China. Email: wuweiyahoo@sohu.com; Xuequan Huang. Treatment Center of Minimally Invasive Intervention and Radioactive Particles, First Affiliated Hospital of the Army Medical University, Chongqing 400038, China. Email: hxuequan@163.com.

Background: Whether preoperative biopsy before radical resection can lead to recurrence and impact patient survival in non-small cell lung cancer (NSCLC) remains controversial. In this study, we carried out a retrospective analysis to determine whether preoperative biopsy can cause disease recurrence and influence disease-free survival (DFS) in patients with stage IA NSCLC.

Methods: Patients diagnosed with stage IA NSCLC (solid nodule) between January 2010 and December 2014 were identified from the databases of 7 Chinese medical centers and divided into two groups: a preoperative computed tomography (CT)-guided needle biopsy (CTNB) plus radical resection group, and a non-CTNB group. The propensity score matching (PSM) method was adopted to balance the observed covariates, and Kaplan-Meier estimates were used for survival analysis. Cox regression was used in a singlefactor analysis to identify the factors affecting DFS in stage IA NSCLC.

Results: After initial screening, 730 patients were enrolled in this study, with 186 and 544 patients in the CTNB group and the non-CTNB group, respectively. After PSM, 186 patients were eventually included in each group. No significant differences in basic clinical features were identified between the two groups $(\mathrm{P}>0.05)$. The rates of recurrence were $17.2 \%$ and $14.0 \%$ in the CTNB and non-CTNB groups $\left(\chi^{2}=0.735\right.$, $\mathrm{P}=0.391)$, respectively. No notable differences in DFS $\left(\chi^{2}=1.895, \mathrm{P}=0.173\right)$ or overall survival (OS, $\chi^{2}=1.785$, $\mathrm{P}=0.182)$ were observed. Lung adenocarcinoma [hazard ratio $(\mathrm{HR}), 0.167, \mathrm{P}=0.001$ ] and lesion size $(>2 \mathrm{~cm})$ (HR, 2.712, $\mathrm{P}=0.000)$ were identified as risk factors for DFS in stage IA NSCLC.

Conclusions: CTNB does not increase the incidence of recurrence in stage IA NSCLC or affect patient survival; therefore, it is not a risk factor for DFS. Lung adenocarcinoma and lesion size are risk factors for DFS.

Keywords: Computed tomography-guided needle biopsy (CTNB); stage IA non-small cell lung cancer (stage IA NSCLC); disease-free survival (DFS) 
Submitted Jul 31, 2020. Accepted for publication Apr 02, 2021.

doi: 10.21037/qims-20-931

View this article at: http://dx.doi.org/10.21037/qims-20-931

\section{Introduction}

Low-dose spiral computed tomography (CT) screening for the high-risk lung cancer population can reduce lung cancer mortality by $20 \%$ (1). Through the application of low-dose spiral CT, more solid pulmonary nodules can be discovered. In China, surgery is the preferred treatment for lung nodules in patients highly suspected with lung cancer (2). In a study of direct surgical resection of 8-20-mm pulmonary nodules, $35 \%$ of nodules were found to be benign postoperatively (3); thus, patients with benign pulmonary nodules may benefit from preoperative biopsy. CT-guided percutaneous biopsy is one of the easiest and fastest methods for the preoperative diagnosis of nodular lung cancer; it has high diagnostic accuracy (4), can provide a decision-making basis for appropriate clinical procedures, and can also relieve the psychological pressure on patients. However, biopsy has also been reported to lead to disease recurrence and may affect patients' long-term survival $(5,6)$. In cases of cancerous lung nodules, whether the percutaneous biopsy process increases the likelihood of recurrence and affects long-term survival remains controversial.

To address this uncertainty, we designed a retrospective cohort study which enrolled patients from multiple centers in China to evaluate whether preoperative biopsy increases the recurrence rate of early-stage non-small cell lung cancer (NSCLC) and affects patient survival. Additionally, we preliminarily investigated the factors influencing recurrence in patients with stage IA NSCLC (solid nodule).

\section{Methods}

\section{Study population}

We collected the clinical information of patients who underwent radical resection and were postoperatively diagnosed with stage IA NSCLC between January 2010 and December 2014 from the databases of 7 medical centers. All data were processed according to the 8 th edition of the Union for International Cancer Control's tumor, node, metastasis (TNM) staging system (7). The following patient information was obtained: sex, age, smoking history, lesion size ( $\leq 2$ vs. $2-3 \mathrm{~cm})$, preoperative biopsy (yes $v s$. no), lesion location (left lung vs. right lung), pathological subtype (adenocarcinoma $v s$. squamous cell lung cancer and other types of NSCLC), and postoperative adjuvant chemotherapy within 6 months (platinum-based chemotherapy regimens). The CT-guided needle biopsy (CTNB) group mainly included patients with atypical imaging features or patients who refused direct surgical resection.

The exclusion criteria were as follows: (I) a postoperative diagnosis of small cell lung cancer; (II) concurrent cancer; (III) a ground-glass nodule; (IV) non-tumor-related death; (V) a surgical approach using wedge resection; (VI) radiofrequency ablation or radiotherapy; (VII) loss to follow-up; and (VIII) an unclear time or location of recurrence. Finally, 730 patients were included in the study (Figure 1).

\section{Evaluation of recurrence and survival}

The patients' CT scan information was reviewed; postoperatively, scans had been conducted at intervals of 6-12 months for a 5-year period. Metastatic lesions were diagnosed based on the occurrence of new lesions, as well as an increased size and number of lesions during the CT follow-up. The first instance of disease recurrence was defined as the endpoint of diseasefree survival (DFS). If CT follow-up data could not be obtained, the time at which metastasis was first discovered (based on the patient's complaint) was considered to be the endpoint of DFS.

\section{Observation indicators}

The incidence of recurrence, DFS, and overall survival (OS) were observed among the patients. DFS was defined as the time from surgery to the first confirmed recurrence event or death due to disease progression. OS was defined as the time from surgery to the last follow-up or tumor-related death. The survival time was measured from the date of surgery to October 30, 2018 (the deadline for the final visit).

\section{Statistical analysis}

All data were described as the means \pm standard deviation for continuous variables and as numbers (percentages) 
Patients with pathologic stage IA lung cancer who underwent curative lung resection between Jan 2010 and Dec $2014(n=794)$

Exclude patients $(n=64)$

-Miss data $(n=27)$

-Small cell lung cancer $(n=10)$

-Dual-source carcinoma $(n=12)$

$-\mathrm{GGO}(\mathrm{n}=4)$

-Non-tumor related death $(n=4)$

-Wedge excision and Non-surgical resection $(n=7)$

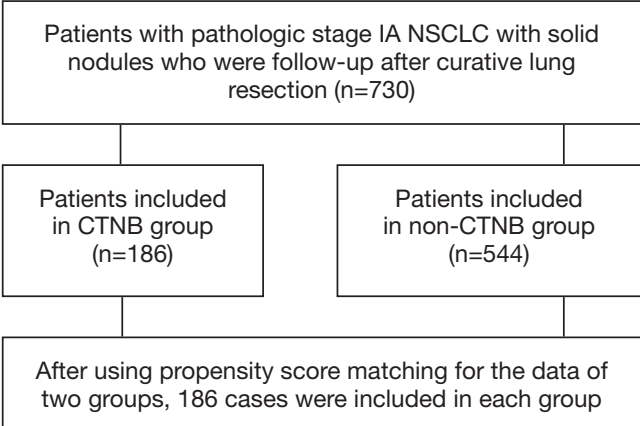

Figure 1 Flowchart showing the patient selection criteria. GGO, ground-glass opacity; NSCLC, non-small cell lung cancer; CTNB, computed tomography-guided needle biopsy.

for categorical variables. Data were compared using the $t$-test for continuous variables, and Pearson's $\chi^{2}$ test or Fisher's exact test for categorical variables. To ensure balance between the groups, propensity score matching (PSM) was carried out to match the patients in both groups (caliper value of 0.05 ). The matched variables included sex, age, smoking history, lesion size ( $\leq 2 v s .2-3 \mathrm{~cm})$, lesion location (left lung vs. right lung), and pathological type (adenocarcinoma $v s$. non-adenocarcinoma). The KaplanMeier method with the log-rank test was used to estimate the difference in time-to-event outcomes between the CTNB and non-CTNB groups using two-sided P values. Cox regression was used in a single-factor analysis to identify the factors affecting DFS in stage IA NSCLC patients. In all analyses, a two-sided $\mathrm{P}$ value $<0.05$ was considered to be an indication of a statistically significant difference. Statistical analyses were performed using SPSS version 25.0 software (SPSS, Chicago, Ill, USA).

\section{Results}

\section{Clinical and pathological characteristics}

Of the 730 patients initially screened, 186 (25.5\%) underwent CTNB as a diagnostic procedure before radical resection and $544(74.5 \%)$ underwent radical resection without biopsy. After radical surgery, all patients were diagnosed with stage IA NSCLC. The baseline characteristics of the patients are summarized in Table 1. The study population comprised 392 males, with an average age of $59.51 \pm 8.85$ years, and 338 females, with an average age of $58.23 \pm 8.79$ years. The mean lesion size was approximately $2.05 \pm 0.65 \mathrm{~cm}(0.4-3 \mathrm{~cm})$. Right lung lesions and left lung lesions accounted for $59.2 \%$ (432/730) and $40.8 \%$ (298/730) of all tumors, respectively. After radical resection of the lung tumors, pathological diagnosis was performed; $80.7 \%(589 / 730)$ of the patients were diagnosed with adenocarcinoma, $18.4 \%$ (134/730) were diagnosed with squamous cell carcinoma, and $0.01 \%(7 / 730)$ were diagnosed with other types of NSCLC, including 68 cases of pT1aN0M0 ( $\leq 1 \mathrm{~cm}), 349$ cases of pT1bN0M0 $(1-2 \mathrm{~cm})$, and 313 cases of pT1cN0M0 $(2-3 \mathrm{~cm})$. In the CTNB group, the biopsy pathology of 1 patient was a false-negative result, and the biopsy pathological types in 4 patients were inconsistent with the postoperative outcomes. The coincidence rate of pathological diagnosis between the 2 diagnostic methods was $97.3 \%$ (181/186). Metastatic sites primarily included the lung, bone, and craniocerebral regions (Table 2).

\section{PSM}

Before PSM, significant differences in the proportion of patients aged $>60$ years and the number of smokers were identified between the CTNB and non-CTNB groups $(\mathrm{P}<0.05)$. Also, a significant difference in the proportion of patients with a lesion size $\leq 2 \mathrm{~cm}$ was found between the groups $(\mathrm{P}<0.05)$. A total of 372 patients were selected using PSM, and the differences in the baseline data between the two groups after PSM were not statistically significant $(\mathrm{P}>0.05$, Table 1).

\section{Recurrence rates and mortality}

Before PSM, the rate of recurrence in the CTNB and nonCTNB groups was $18.8 \%$ (35/186) and 8.6\% (47/544), respectively. Therefore, the incidence of recurrence in the CTNB group was significantly higher than that in the nonCTNB group $\left(\chi^{2}=11.014, \mathrm{P}=0.000\right)$. After PSM, the rate of recurrence in the CTNB and non-CTNB groups was $17.2 \%$ and $14.0 \%\left(\chi^{2}=0.735, \mathrm{P}=0.391\right)$, respectively (Table 2$)$.

Before PSM, the mortality rate in the CTNB and nonCTNB groups was $11.8 \%(22 / 186)$ and $4.2 \%(23 / 544)$, respectively. Therefore, the mortality rate in the CTNB 
Table 1 Clinical characteristics of the patients

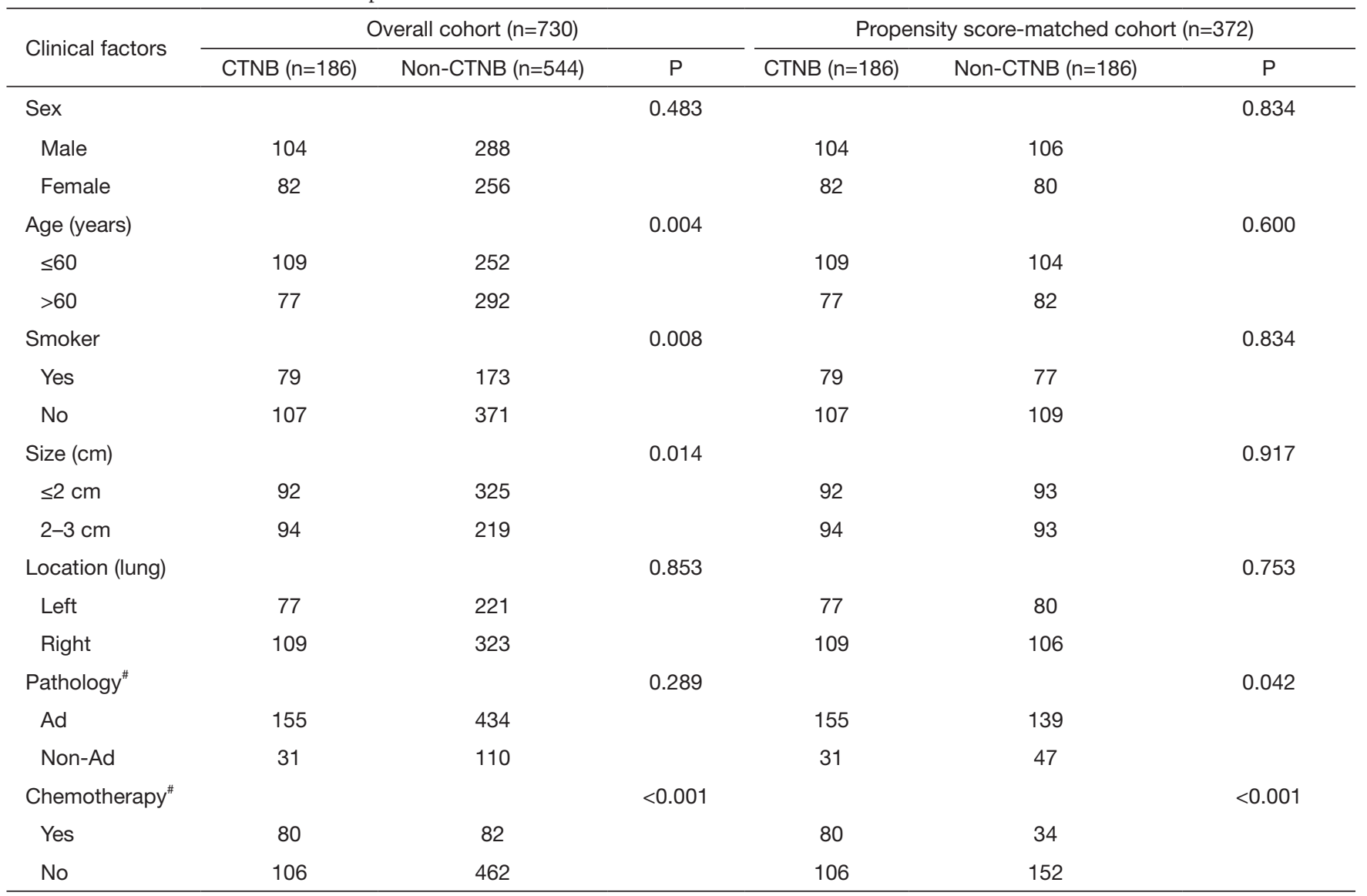

\#, it is not a variable of propensity score matching. Ad, adenocarcinoma; non-Ad, non-adenocarcinoma; CTNB, computed tomographyguided needle biopsy.

group was significantly higher than that in the non-CTNB group $\left(\chi^{2}=13.841, \mathrm{P}=0.000\right)$. After PSM, the mortality rate in the CTNB and non-CTNB groups was $11.8 \%(22 / 186)$ and $8.6 \%(16 / 186)$, respectively $\left(\chi^{2}=1.055, \mathrm{P}=0.305\right)$.

\section{Survival analysis}

The DFS $\left(\chi^{2}=13.131, \mathrm{P}=0.000\right)$ and OS $\left(\mathrm{OS}, \chi^{2}=15.657\right.$, $\mathrm{P}=0.000)$ in the CTNB group before PSM were both markedly shorter than those in the non-CTNB group. After PSM, the median survival time was 62 months (interquartile range, $45,66.7)$. The OS rates at 1,3 , and 5 years were $99.7 \%, 95.4 \%$, and $89.8 \%$, respectively. There was no significant difference in DFS $\left(\chi^{2}=1.895, \mathrm{P}=0.173\right)$ or OS (OS, $\left.\chi^{2}=1.785, \mathrm{P}=0.182\right)$ between the CTNB and non-CTNB groups after matching (Figures 2,3).

\section{Univariate and multivariate analyses of recurrence}

Before PSM, the univariate analysis of time to recurrence revealed significant differences in pathological type $(\mathrm{P}=0.034)$, tumor size $(\mathrm{P}=0.000)$, and preoperative biopsy $(\mathrm{P}=0.000)$ between the groups (Table 3). Statistical analysis showed that when all factors were included in the multivariate analysis, preoperative biopsy [hazard ratio (HR), 1.854, $\mathrm{P}=0.007$ ], lung adenocarcinoma (HR, 0.340, $\mathrm{P}=0.003)$, and tumor size $(\mathrm{HR}, 2.425, \mathrm{P}=0.000)$ were risk factors for DFS.

After PSM, the univariate analysis of DFS showed statistically significant differences in tumor size $(\mathrm{P}=0.002)$ and pathological type $(\mathrm{P}=0.003)$ between the groups. The multivariate analysis of DFS after PSM showed that lung adenocarcinoma (HR, 0.167, $\mathrm{P}=0.001)$ and tumor size (HR, 2.712, $\mathrm{P}=0.000$ ) were risk factors for DFS (Table 3). 
Table 2 Recurrence in patients with resected stage IA NSCLC

\begin{tabular}{|c|c|c|c|c|c|c|}
\hline Variables & \multicolumn{3}{|c|}{ Overall cohort $(n=730)$} & \multicolumn{3}{|c|}{ Propensity score-matched cohort $(n=372)$} \\
\hline Recurrence, n (\%) & $35(18.8)$ & $47(8.6)$ & 0.000 & $32(17.2)$ & $26(14.0)$ & 0.391 \\
\hline Brain & $6(3.2)$ & $3(0.6)$ & & $6(3.2)$ & $2(1.1)$ & \\
\hline Lung & $14(7.5)$ & $10(1.8)$ & & $11(5.9)$ & $6(3.2)$ & \\
\hline Lymph node & $2(1.1)$ & $10(1.8)$ & & $2(1.1)$ & $6(3.2)$ & \\
\hline Pleural & $1(0.5)$ & $2(0.4)$ & & $1(0.5)$ & $0(0.0)$ & \\
\hline Adrenal gland & $1(0.5)$ & $0(0.0)$ & & $1(0.5)$ & $0(0.0)$ & \\
\hline Eyeball & $1(0.5)$ & $0(0.0)$ & & $1(0.5)$ & $0(0.0)$ & \\
\hline
\end{tabular}

\#, multisystem transfer. NSCLC, non-small cell lung cancer; CTNB, computed tomography-guided needle biopsy.

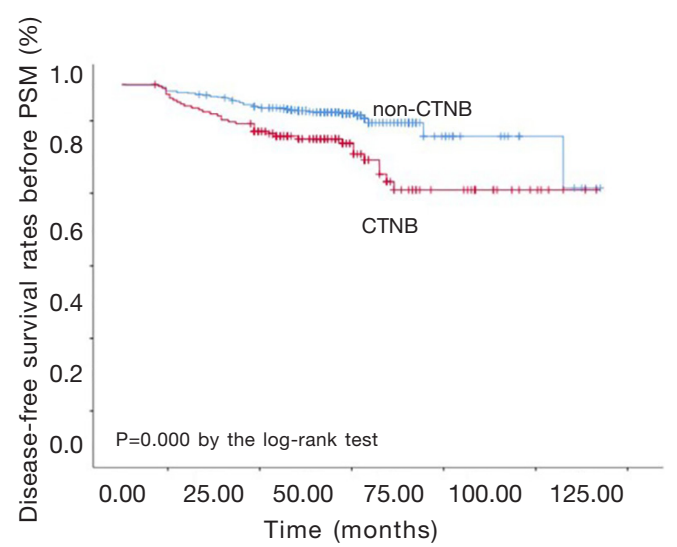

Figure 2 Comparison of disease-free survival (DFS) between the computed tomography-guided needle biopsy (CTNB) and non-CTNB groups. Kaplan-Meier analysis of the time to total recurrence between the CTNB and non-CTNB groups.

\section{Discussion}

Compared with transbronchial lung biopsy, percutaneous biopsy has higher accuracy for diagnosing lung nodules (8). The diagnostic accuracy of biopsy for sub-centimeter lung nodules can reach $88-95 \%$ (9). Our study showed that the diagnostic accuracy of pathological biopsy for benign and malignant nodules reached $99.5 \%$ (185/186). Moreover, comparing the results of our team's previous study (4) with those of the study by Tanner et al. (3), no significant

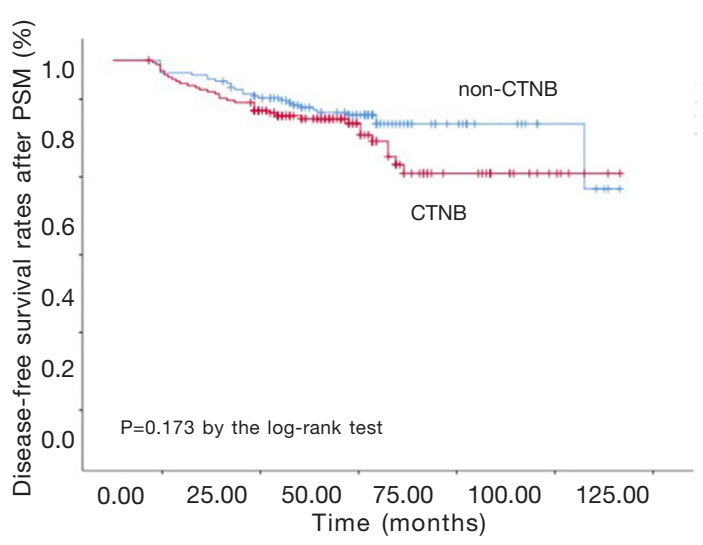

Figure 3 Comparison of disease-free survival (DFS) between the computed tomography-guided needle biopsy (CTNB) and non-CTNB groups. Kaplan-Meier analysis of the time to total recurrence after propensity score matching between the CTNB and non-CTNB groups.

differences in the two methods (biopsy vs. surgery) exist in the observation rates for benign and malignant pulmonary nodules $\left(\chi^{2}=0.568, \mathrm{P}=0.451\right)$. Hemorrhage and pneumothorax are the common complications of solid nodule percutaneous biopsy (10). With the development of percutaneous biopsy instruments and advances in surgical techniques, the incidence of both common and rare surgical complications can be effectively controlled.

Puncture biopsy needle track implantation is a clinical issue that cannot be ignored. Studies have reported the rate 
Table 3 Univariate and multivariable analysis of predictors of DFS

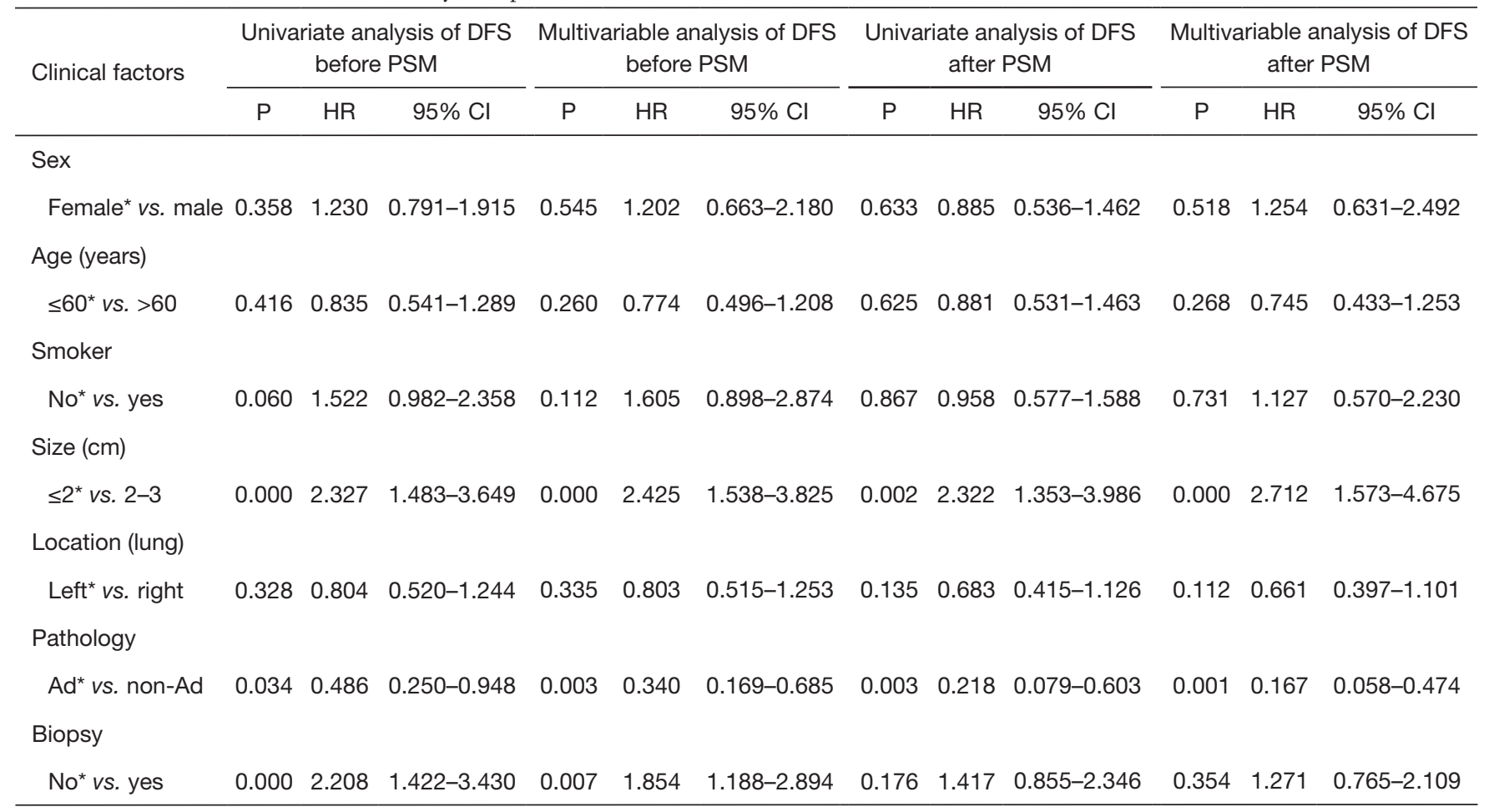

*, baseline reference. HR, hazard ratio; DFS, disease-free survival; PSM, propensity score matching; Ad, adenocarcinoma; non-Ad, nonadenocarcinoma.

of needle track implantation with osteosarcoma biopsy to be as high as $20 \%(11,12)$. Some reports have also described needle track implantation with lung cancer biopsy, and this difference in the incidence of needle metastasis may be attributable to different tumor types. Needle-track metastasis may be caused by the presence of tumor cells in the needle track after biopsy or biopsy resulting in increased messenger RNA levels of tumor biomarkers. However, tumor cells are eliminated by the autoimmune system. Clinically significant lesions due to needle track implantation are present in a small number of cases, and the incidence of clinical needle track implantation is approximately $0.016-0.018 \%(13-18)$. In this study, no needle-track metastasis was found in the CTNB group. After PSM, the rate of pulmonary recurrence and metastasis in the CTNB group was similar to that in the non-CTNB group, although the rate in the CTNB group was slightly higher. However, we did not document the specific sites of pulmonary metastases, and the lack of in-depth analysis was a shortcoming of this study.

With the continuous improvement of treatment methods, especially targeted and immunotherapeutic applications, patients with recurrence can achieve better OS. Therefore, we primarily used DFS as the study endpoint. The CTNB group in this study had significantly increased recurrence rates before PSM, and the DFS was also significantly shortened. To standardize the baseline data of the two groups, the PSM method was used. After PSM, no significant differences were noted between the recurrence rates of the two groups, or in DFS or OS. Therefore, we believe that preoperative biopsy does not promote recurrence or affect the DFS or OS of patients, which reinforces the results of previous retrospective research (19-22). However, other retrospective studies have reported that percutaneous biopsy may promote recurrence and metastasis of early-stage lung cancer and is extremely likely to affect the long-term survival of patients $(5,23)$. At the same time, our study showed that adjuvant chemotherapy in the biopsy group did not have a DFS benefit for patients $\left(\chi^{2}=0.001, \mathrm{P}=0.970\right)$ and that adjuvant chemotherapy is not recommendable for patients after percutaneous biopsy. However, further prospective studies are needed to confirm these findings.

Recurrence following radical resection of early-stage 
NSCLC remains poorly understood and is multifactorial in nature. Multivariate analysis before PSM demonstrated that preoperative biopsy, lung adenocarcinoma, and tumor size were factors affecting the DFS of patients. The influencing factors of DFS in stage IA NSCLC were also analyzed after PSM. After matching the two datasets, we found that tumor size and lung adenocarcinoma were the influencing factors of DFS. Significant differences in DFS were observed between patients with tumor sizes $\leq 2 \mathrm{~cm}$ and those with tumor sizes of $2-3 \mathrm{~cm}\left(\log -\mathrm{rank} / \chi^{2}=9.960\right.$, $\mathrm{P}=0.002$ ), which is consistent with the results of Zhang et al. (24). In the current multivariate analysis, tumor size $(>2 \mathrm{~cm})$ was again confirmed to be an important predictor of recurrence following resection of stage IA NSCLC. Studies have shown that non-squamous cell carcinoma is a risk factor for the prognosis of NSCLC (25). The present study categorized the pathological types into adenocarcinoma and non-adenocarcinoma, with the latter comprising squamous cell carcinoma and NSCLC with other pathological types. Lung adenocarcinoma type was also found to be a risk factor for the prognosis of NSCLC, with the DFS of patients with adenocarcinoma being statistically significantly shorter than that of non-adenocarcinoma patients $\left(\log -\mathrm{rank} / \chi^{2}=10.428, \mathrm{P}=0.001\right)$.

\section{Limitations}

This study has some limitations that should be noted. Firstly, this study did not provide accurate and complete data for analyses of whether or not the lesions were subpleural nodules, the degree of pathological differentiation, the extent of infiltration, or the specific specifications of the percutaneous biopsy needles, which is the primary drawback of this study. Secondly, the design of retrospective studies has inherent flaws, which make further validation by prospective studies necessary. Thirdly, in the CTNB and non-CTNB groups, bias existed in the patient selection; although, we did attempt to reduce this bias through PSM analysis. Fourthly, this study utilized the overall recurrence rate, and metastatic sites were not included in the analyses. Finally, although we used DFS as the primary endpoint, the small number of DFS outcomes reduces the reliability of the multivariate analysis results.

However, this research also provides valuable information. In our Chinese study population, we found that the use of preoperative biopsy in patients with stage IA NSCLC undergoing radical resection of lung cancer did not increase the incidence of recurrence and did not affect patient survival. Moreover, this study is the first multicenter retrospective cohort study on preoperative biopsy for early lung cancer in China and provides guidance for future clinical practice.

\section{Conclusions}

CTNB performed in patients with stage IA NSCLC (solid nodules) does not increase the incidence of recurrence or affect patient survival, and is not a risk factor for DFS. Therefore, this procedure can be safely used to diagnose stage IA NSCLC. Lung adenocarcinoma and lesion size are risk factors for the DFS of patients with stage IA NSCLC (solid nodules). However, prospective randomized trials are needed in the future to confirm our findings.

\section{Acknowledgments}

Funding: This study was supported by the Medical Research Projects of Chongqing Science and Technology Bureau and Health and Health Committee (2021MSXM342), and the Technological Innovation and Application Development in Chongqing (Science and Technology Poverty Alleviation Project (CSTC2019jscx-KJFP0005).

\section{Footnote}

Conflicts of Interest: All authors have completed the ICMJE uniform disclosure form (available at http://dx.doi. org/10.21037/qims-20-931). The authors have no conflicts of interest to declare.

Ethical Statement: The authors are accountable for all aspects of the work in ensuring that questions related to the accuracy or integrity of any part of the work are appropriately investigated and resolved. The retrospective study was conducted in accordance with the Declaration of Helsinki (as revised in 2013), and was approved by the Ethics Committee of the First Affiliated Hospital of the Army Medical University (IRB No. KY201905). Informed consent for the use of medical data was waived as the patient information was anonymized and de-identified prior to analysis.

Open Access Statement: This is an Open Access article distributed in accordance with the Creative Commons Attribution-NonCommercial-NoDerivs 4.0 International License (CC BY-NC-ND 4.0), which permits the non- 
commercial replication and distribution of the article with the strict proviso that no changes or edits are made and the original work is properly cited (including links to both the formal publication through the relevant DOI and the license). See: https://creativecommons.org/licenses/by-nc-nd/4.0/.

\section{References}

1. National Lung Screening Trial Research Team, Aberle DR, Adams AM, Berg CD, Black WC, Clapp JD, Fagerstrom RM, Gareen IF, Gatsonis C, Marcus PM, Sicks JD. Reduced lung-cancer mortality with lowdose computed tomographic screening. N Engl J Med 2011;365:395-409.

2. Zhou Q, Fan Y, Wang Y, Qiao Y, Wang G, Huang Y, Wang X, Wu N, Zhang G, Zheng X, Bu H, Li Y, Wei S, Chen L, Hu C, Shi Y, Sun Y. China National Lung Cancer Screening Guideline with Low-dose Computed Tomography (2018 version). Zhongguo Fei Ai Za Zhi 2018;21:67-75.

3. Tanner NT, Aggarwal J, Gould MK, Kearney P, Diette G, Vachani A, Fan KC, Silvestri GA. Management of pulmonary nodules by community pulmonologists: a multicenter observational study. Chest 2015;148:1405-14.

4. Yang L, Zhang Q, Bai L, Li TY, He C, Ma QL, Li LS, Huang XQ, Qian GS. Assessment of the cancer risk factors of solitary pulmonary nodules. Oncotarget 2017;8:29318-27.

5. Xi Y, Fan J, Che D, Zhai K, Ren T, Feng X, Shang L, Hu J, Yu Y, Meng Q. Distant Metastasis and Survival Outcomes after Computed Tomography-Guided Needle Biopsy in Resected Stage I-III Non-Small Cell Lung Cancer. J Cancer 2017;8:3356-61.

6. Kashiwabara K, Semba H, Fujii S, TsumuraS. Preoperative percutaneous transthoracic needle biopsy increased the risk of pleural recurrence in pathological stage I lung cancer patients with sub-pleural pure solid nodules. Cancer Invest 2016;34:373-7.

7. Goldstraw P, Chansky K, Crowley J, Rami-Porta R, Asamura H, Eberhardt WE, Nicholson AG, Groome P, Mitchell A, Bolejack V; International Association for the Study of Lung Cancer Staging and Prognostic Factors Committee, Advisory Boards, and Participating Institutions; International Association for the Study of Lung Cancer Staging and Prognostic Factors Committee Advisory Boards and Participating Institutions. The IASLC Lung Cancer Staging Project: Proposals for Revision of the TNM Stage Groupings in the Forthcoming (Eighth) Edition of the TNM Classification for Lung Cancer. J Thorac Oncol 2016;11:39-51.

8. Han Y, Kim HJ, Kong KA, Kim SJ, Lee SH, Ryu YJ, Lee JH, Kim Y, Shim SS, Chang JH. Diagnosis of small pulmonary lesions by transbronchial lung biopsy with radial endobronchial ultrasound and virtual bronchoscopic navigation versus CT-guided transthoracic needle biopsy: A systematic review and meta-analysis. PLoS One 2018;13:e0191590.

9. Wallace MJ, Krishnamurthy S, Broemeling LD, Gupta S, Ahrar K, MorelloJr FA, Hicks ME. CT-guided percutaneous fine-needle aspiration biopsy of small $(<$ or =1-cm) pulmonary lesions. Radiology 2002;225:823-8.

10. Tavare AN, Hare SS, Miller FNA, Hammond CJ, Edey A, Devaraj A. A survey of UK percutaneous lung biopsy practice: current practices in the era of early detection, oncogenetic profiling, and targeted treatments. Clin Radiol 2018;73:800-9.

11. Turkoz KH, Erol B, Seven IE. Tumor cell seeding in the biopsy tract and its clinical significance in osteosarcomas. J Surg Oncol 2018;118:1335-40.

12. Zhu J, Qu Y, Wang X, Jiang C, Mo J, Xi J, Wen Z. Risk factors associated with pulmonary hemorrhage and hemoptysis following percutaneous CT-guided transthoracic lung core needle biopsy: a retrospective study of 1,090 cases. Quant Imaging Med Surg 2020;10:1008-20.

13. Richardson CM, Pointon KS, Manhire AR, Macfarlane JT. Percutaneous lung biopsies: a survey of UK practice based on 5444 biopsies. Br J Radiol 2002;75:731-5.

14. Tomiyama N, Yasuhara Y, Nakajima Y, Adachi S, Arai Y, Kusumoto M, Eguchi K, Kuriyama K, Sakai F, Noguchi M, Murata K, Murayama S, Mochizuki T, Mori K, Yamada K. CT-guided needle biopsy of lung lesions: a survey of severe complication based on 9783 biopsies in Japan. Eur J Radiol 2006;59:60-4.

15. Shyamala K, Girish HC, Murgod S. Risk of tumor cell seeding through biopsy and aspiration cytology. J Int Soc Prev Community Dent 2014;4:5-11.

16. Struve-Christensen E. Iatrogenic dissemination of tumour cells. Dissemination of tumour cells along the needle track after percutaneous, transthoracic lung biopsy. Dan Med Bull 1978;25:82-7.

17. Steeg PS. Tumor metastasis: mechanistic insights and clinical challenges. Nat Med 2006;12:895-904.

18. Hu XC, Chow LW. Fine needle aspiration may shed breast cells into peripheral blood as determined by RT- 
PCR. Oncology 2000;59:217-22.

19. Wang T, Luo L, Zhou Q. Risk of Pleural Recurrence in Early Stage Lung Cancer Patients after Percutaneous Transthoracic Needle Biopsy: A Meta-analysis. Sci Rep 2017;7:42762.

20. Moon SM, Lee DG, Hwang NY, Ahn S, Lee H, Jeong BH, Choi YS, Shim YM, Kim TJ, Lee KS, Kim H, Kwon OJ, Lee KJ. Ipsilateral pleural recurrence after diagnostic transthoracic needle biopsy in pathological stage I lung cancer patients who underwent curative resection. Lung Cancer 2017;111:69-74.

21. Wisnivesky JP, Henschke CI, Yankelevitz DF. Diagnostic percutaneous transthoracic needle biopsy does not affect survival in stage I lung cancer. Am J Respir Crit Care Med 2006;174:684-8.

22. Hu C, Jiang J, Li Y, Zhang C, Zhang W, Jiang H, Gao Y, Zhuang W, Lei K, T ang Y, Wan R. Recurrence risk after

Cite this article as: $\mathrm{He} \mathrm{C}$, Yu H, Li C, Zhang X, Huang Z, Liu M, Tong L, Zhu J, Wu W, Huang X. Recurrence and disease-free survival outcomes after computed tomographyguided needle biopsy in stage IA non-small cell lung cancer patients in China: a propensity score matching analysis. Quant Imaging Med Surg 2021;11(8):3472-3480. doi: 10.21037/qims-20931 preoperative biopsy in patients with resected early-stage non-small-cell lung cancer: a retrospective study. Cancer Manag Res 2018;10:1927-34.

23. Fan J, Zhai K, Ren T, et al. Distant Metastasis and Survival Outcomes after Computed Tomography-guided Needle Biopsy in Stage I-II Non-small Cell Lung Cancer. Zhongguo Fei Ai Za Zhi 2017;20:187-91.

24. Zhang Y, Sun Y, Xiang J, Zhang Y, Hu H, Chen H. A clinicopathologic prediction model for postoperative recurrence in stage Ia non-small cell lung cancer. J Thorac Cardiovasc Surg 2014;148:1193-9.

25. Okada M, Nishio W, Sakamoto T, Harada H, Uchino K, Tsubota N. Long-term survival and prognostic factors of five-year survivors with complete resection of nonsmall cell lung carcinoma. J Thorac Cardiovasc Surg 2003;126:558-62. 